\title{
CONSIDERAZIONI CONCLUSIVE
}

\author{
ENRICO DECLEVA (*)
}

Siamo arrivati alla conclusione di questa seduta pomeridiana dedicata alle responsabilità istituzionali di Paolo Mantegazza: e quindi ai dieci anni di presidenza della Facoltà di Medicina, dal 1974 al 1984 e ai diciassette anni di rettorato dal 1984 al 2001, senza dimenticare gli impegni successivi svolti a salvaguardia e a difesa della ricerca scientifica all'interno della Fondazione Cariplo, ricordati dal presidente Guzzetti.

Credo che sia mio compito ringraziare a questo punto, a nome di tutti, gli oratori che si sono susseguiti per l'impegno, l'efficacia, la forte carica di partecipazione affettiva che hanno trasfuso nei loro interventi, richiamando alla nostra memoria i vari passaggi dell'opera di Paolo Mantegazza, le scelte compiute, gli effetti sostanziali e di lungo periodo che esse hanno avuto, e non solo per l'Università degli Studi di Milano. $\mathrm{Ma}$ ricordandoci anche, e soprattutto, le spinte di fondo, le radici umane e religiose che hanno ispirato la sua azione, connotandola in una maniera del tutto particolare.

Non sono stati decenni facili per la vita universitaria, nazionale e milanese, quelli nei quali Paolo Mantegazza si è trovato ad avere responsabilità via via più rilevanti. Molti dei presenti ricordano la Statale dei primi anni '60: un ateneo con poco più di 10 mila iscritti: diventati 20 mila alla fine del decennio e poi cresciuti senza soste fino ai 63 mila del 1978-79 (che sarebbero diventati 95 mila negli anni '90). Una crescita alla quale non aveva corrisposta quella degli spazi, delle risorse, del numero dei docenti, delle dotazioni di personale tecnicoamministrativo.

(*) Istituto Lombardo Accademia di Scienze e Lettere. Università degli Studi di Milano, Italia. E-mail: enrico.decleva@unimi.it 
Fu solo a partire dal piano edilizio universitario del 1976-1981 che si cominciò a riconoscere l'effettivo peso della Statale nel sistema universitario nazionale, e fu da allora che iniziarono, in effetti, sotto il rettorato di Giuseppe Schiavinato, le prime realizzazioni. Ma sempre inseguendo una situazione che continuava a evolvere e a determinare nuove difficoltà. Costringendo sempre a operare all'insegna dell'emergenza e dei rimedi da trovare in itinere. Rimedi che, nella situazione altrimenti ingovernabile della Facoltà di Medicina, furono identificati dall'allora preside Mantegazza, come è stato ricordato da Guido Coggi, nella realizzazione degli otto poli ospedalieri riconosciuti dalla Regione Lombardia nel 1977, di cui cinque a Milano. E varrà la pena di ricordare come scegliendo quella strada si seguissero in realtà, per alcuni aspetti, le orme del fondatore dell'Università milanese, Luigi Mangiagalli, che proprio grazie allo strumento convenzionale era riuscito a dare progressivamente vita a una Facoltà medica di prim'ordine senza disporre in partenza di un policlinico universitario.

Ma il balzo improvviso a università di massa riguardava e metteva in estrema difficoltà tutto l'Ateneo, non solo la Facoltà di Medicina e Chirurgia. E' stato ricordato il caso-limite della Facoltà di Giurisprudenza, giunta ad avere nel 1984 un rapporto docenti/studenti di 1:190.

Non era facile, in una simile situazione, tener fede all'ambizione di restare un ateneo di primo livello quanto a ricerca e qualità della didattica. Oltretutto, dovendo contemporaneamente fronteggiare i periodici ritorni di contestazione e di conflittualità, compresa, ancora nel 1990, una lunga occupazione dell'aula magna, conclusasi con la sua completa devastazione nella sostanziale indifferenza delle autorità, come è stato ricordato.

L'impresa di Mantegazza, rievocata da Giampiero Sironi, è stata di rispondere alla complessità delle sfide, con le realizzazioni molto numerose avviate e completate entro il 2001 (quando ha lasciato il Rettorato) e successivamente: e qualcuna è ancora da concludere (il completo trasferimento di Medicina veterinaria a Lodi). La scelta strategica più rilevante è stata quella, ampiamente richiamata da Marcello Fontanesi, delle "gemmazioni" da cui sono nate le Università pubbliche di MilanoBicocca e dell'Insubria e quella privata San Raffaele-Vita e salute. Una scelta che, come sappiamo bene, ha inciso in profondità sul panorama universitario cittadino e regionale. E che ben difficilmente sarebbe giunta a effetto se al timone della Statale non ci fosse stato Mantegazza. 
Quelle compiute nell'era Mantegazza furono scelte rese possibili dai maggiori stanziamenti ordinari (che, almeno in parte, cominciavano a riconoscere sulla base di indicatori oggettivi il peso della Statale) e da stanziamenti straordinari, in una fase (credo che vada detto) in cui da parte della politica nazionale l'università costituiva un settore di relativo impegno, e non, come sarebbe accaduto in seguito, di disimpegno o peggio. Ma giovarono non poco anche i rapporti che Mantegazza seppe costruire con ministri e direttori generali, e che certo giovarono a una migliore considerazione degli interessi e delle aspirazioni dell'ateneo.

Le risorse pubbliche non sarebbero d'altro canto bastate, per quel che riguarda in particolare la situazione della Statale, senza la scelta di alzare la contribuzione studentesca: una scelta peraltro compiuta e fatta approvare da Mantegazza, stabilendo che i relativi incrementi fossero in larga misura indirizzati al potenziamento della didattica, consentendo in particolare ad alcune Facoltà realizzazioni significative e altrimenti impossibili.

Sicuramente tra i rettori che più hanno influito sulla vita dell'Ateneo, Paolo Mantegazza non è mai stato percepito un "uomo solo al comando". E' stato, come ha ricordato poco fa Antonio Padoa Schioppa, davvero il "rettore di tutti", così come si era impegnato a essere quando fu eletto per la prima volta, rispondendo alla provocazione di un giornalista, e sostenendo che sarebbe stato il rettore non di Medicina, ma, appunto, "di tutti". E fu in effetti quello il criterio da lui mai abbandonato alla testa del Senato accademico: tenendo conto delle esigenze di tutte le Facoltà e coinvolgendo ampiamente i loro Presidi sia nei processi decisionali sia poi, con ampie deleghe, nelle fasi realizzative. Senza privilegiare nessuno; cercando e quasi sempre trovando, nella distribuzione delle risorse o nei momenti di contrasto, i punti di possibile equilibrio e conciliazione.

$\mathrm{Ma}$, come è stato ricordato, Mantegazza fu rettore di tutti ben al di là del vecchio Senato dei Presidi; lo fu rispetto all'intera comunità universitaria: una comunità, nel caso della Statale, con la sua storia e la sua configurazione, estremamente variegata e composita, in tutti i sensi. Uno dei meriti di Mantegazza è stato quello di guardare alle persone e al loro lavoro universitario, non allo schieramento o all'ideologia. Comportandosi di conseguenza davanti a richieste o proposte, cercando sempre di accoglierle, senza pregiudiziali o partiti presi. Con una presenza costante e quotidiana in sede. Ricevendo tutti: e riducendo al minimo i tempi di attesa. Ascoltando e venendo incontro nelle richieste. 
Magari equilibrando con fondi rettorali situazioni di singoli in difficoltà con il rispettivo direttore di Dipartimento. Intervenendo nei momenti più problematici anche sul piano personale: sia che si trattasse di docenti, sia che le difficoltà riguardassero il personale tecnico o amministrativo. E sempre con la massima discrezione. Come un buon padre di famiglia: di una famiglia molto vasta e molto larga. Avendo una preoccupazione prioritaria e costante per gli studenti e le loro esigenze, come ci è stato così bene illustrato dall'arcivescovo Brugnaro. Non potendo impedire i periodici ritorni di prevaricazioni e tensioni da parte di specifici gruppi, specie in un luogo simbolico come la sede di via Festa del Perdono, ma evitando situazioni che alimentassero la protesta e ricercando sempre soluzioni e vie d'uscita ragionevoli.

Paolo Mantegazza è stato un elemento di equilibrio anche all'interno del sistema universitario lombardo, come ha mostrato quale presidente del relativo Comitato regionale, manifestando anche in tale ruolo la sua saggezza, il suo grande buon senso, il suo spirito di conciliazione, evitando e superando i contrasti e le contraddizioni che hanno non di rado caratterizzato la gestione di quell'organismo in altre parti della Penisola.

Davvero possiamo dirlo, dopo quanto abbiamo ascoltato, a conferma di quanto ci è rimasto impresso nella memoria: gli anni della presidenza di Facoltà e poi quelli del rettorato di Mantegazza hanno avuto una portata centrale nella storia della Statale e dell'intero sistema universitario milanese e lombardo. Dandoci un'ennesima testimonianza di quanto possano contare le qualità e l'impegno delle persone quando siano all'altezza delle responsabilità assunte. 